\title{
Brain Pharmacokinetics and Tissue Distribution In Vivo of Fluvoxamine and Fluoxetine by Fluorine Magnetic Resonance Spectroscopy
}

Nicolas R. Bolo, Ph.D., Yann Hodé, M.D., Jean-François Nédélec, Ph.D., Eric Lainé, M.D., Gabrielle Wagner, Ph.D., and Jean-Paul Macher, M.D.

This investigation of fluvoxamine and fluoxetinenorfluoxetine distributions in vivo at steady-state and of quantitative kinetics in brain and plasma after drug therapy interruption was performed by fluorine nuclear magnetic resonance spectroscopy $\left({ }^{19} F M R S\right)$, spectroscopic imaging (MRSI), and plasma HPLC on 12 subjects treated for depression. MRSI suggests a homogeneous distribution of ${ }^{19} \mathrm{~F}$ MRS visible fluvoxamine mainly in brain. Fluvoxamine steady-state brain concentrations $(12 \pm 5 \mu M ; \mathrm{n}=13)$ and brain-to-plasma concentration ratios $(10 \pm 2 ; \mathrm{n}=12)$ were similar to those of combined fluoxetine-norfluoxetine (CFnorfluoxetine) $(13 \pm 6 \mu \mathrm{M} ; \mathrm{n}=4$ and $10 \pm 6 ; \mathrm{n}=4)$.
Fluvoxamine brain elimination half-life ( $79 \pm 24$ hours; $\mathrm{n}=4)$ was significantly shorter than that of CF-norfluoxetine (382 \pm 48 hours; $\mathrm{n}=2)$. Fluvoxamine brain-to-plasmahalf-life-ratio was $2.2 \pm 0.3(\mathrm{n}=4)$, contrarily to CF-norfluoxetine $(1.0 \pm 0.3 ; \mathrm{n}=2)$. This study shows that quantitative pharmacokinetics in target organs by ${ }^{19} F$ MRS in vivo should prove useful for understanding and investigating outcome of treatment modifications and side effects.

[Neuropsychopharmacology 23:428-438, 2000] (C) 2000 American College of Neuropsychopharmacology. Published by Elsevier Science Inc.
KEY WORDS: Fluorine MRS; Magnetic resonance spectroscopy; Fluvoxamine; Fluoxetine; Pharmacokinetics; Brain; CNS; Drug tissue distribution

Pharmacodynamic studies mostly rely on plasma kinetics alone because brain concentrations are not easily measured. However, concentrations in the the central nervous system (CNS) should be of more interest for understanding the behavioral effects of psychotropic drugs which are expected to be related to their levels in the target organ, i.e., the brain.

From the FORENAP Center For Research in Neuroscience And Neuropsychiatry, Magnetic Resonance Unit, Rouffach, France.

Address correspondence to: Dr. Nicolas R. Bolo, FORENAP Center for Research in Neuroscience and Neuropsychiatry, Magnetic Resonance Unit, 27 rue du 4 RSM, F-68250 Rouffach, France.

Received November 5, 1999; revised March 7, 2000; accepted March 16, 2000.
Use of radioisotopes in positron emission tomography (PET) or single photon emission computerized tomography (SPECT) studies may provide a way to estimate the receptor occupancy, but these techniques present major disadvantages because of the inherent hazard related to the radioactivity of the tracer. Repeated studies are limited in order to limit radioactive exposure, thus medium or long term kinetic studies by these techniques are excluded. On the contrary, nuclear magnetic resonance (MR) techniques which have been shown to be harmless to the subject under repetitive recording conditions may be of great interest for estimating tissue concentrations of drugs. Indeed, for some drugs that have one or several fluorine atoms in their active structure, no specific labeling is required since the $100 \%$ naturally abundant fluorine-19 nucleus $\left({ }^{19} \mathrm{~F}\right)$ constitutes a natural non-radioactive, stable label of the 
drug detectable by ${ }^{19} \mathrm{~F}$ magnetic resonance spectroscopy (MRS). Thus ${ }^{19} \mathrm{~F}$ MRS provides a means to detect signal from the drug in the tissues in vivo (Durst et al. 1990; Komoroski et al. 1990) and also to estimate drug concentrations in vivo (Renshaw et al. 1992; Karson et al. 1992) when a calibration method is applied to the measurement. This is the case in particular for the two antidepressant selective serotonin reuptake inhibitors (SSRI) fluvoxamine and fluoxetine, which are widely prescribed for treatment of depression, affective or mood disorders, and for obsessive compulsive disorder.

It has also been shown that brain drug kinetics during treatment modifications such as progressive withdrawal (Bolo et al. 1996) and abrupt withdrawal or uptake (Strauss et al. 1997, 1998) may be obtained in this way. However, at the present time little is known about the distribution at steady state of SSRIs in tissues in vivo and their kinetics in target or peripheral tissues. More knowledge on target and peripheral tissue distribution and kinetics should be useful to explain central treatment or secondary effects and could help the clinician for choice of treatment and treatment planning. In this preliminary study, it was our objective to investigate and compare the distribution of the SSRIs fluvoxamine and fluoxetine in tissues and the withdrawal kinetics in brain and in plasma.

\section{MATERIALS AND METHODS}

\section{Subject Population}

This paper reports results from 12 subjects suffering from depression. The study was approved by the CCPPRB Alsace II Ethics Committee, France. Written informed consent was obtained from all subjects prior to participation. All subjects included in the study underwent a detailed psychiatric interview and were under SSRI treatment for a major depressive episode. The DSM-IV criteria for major depressive disorder was clearly met for 11 of the subjects. For one of the subjects, the major depressive episode concurred with an associated diagnosis of generalized anxiety disorder (DSM-IV).

The average age for the eight women and four men who participated was $48 \pm 8$ years and ranged from 36 to 67 years. The average age for the eight subjects treated with fluvoxamine (five women and three men) was $49 \pm 10$ years and ranged from 36 to 67 years. The average age for the four subjects treated with fluoxetine (three women and one man) was $47 \pm 4$ years and ranged from 42 to 50 years. No significant age difference was found between the fluvoxamine and fluoxetine groups or between the men and women subgroups within each treatment group.

Daily fluvoxamine doses ranged from 100 to $300 \mathrm{mg}$ (one subject on $100 \mathrm{mg}$, one subject on $200 \mathrm{mg}$, one sub- ject on $250 \mathrm{mg}$, four subjects on $300 \mathrm{mg}$, and one subject with two treatment periods on $200 \mathrm{mg}$ and $300 \mathrm{mg}$ ). Duration of fluvoxamine treatment ranged from one to 12 months (two subjects for one month, two subjects for three months, one subject for seven months, two subjects for 12 months, and one subject for 12 months on $300 \mathrm{mg}$, and for 3 months on $200 \mathrm{mg}$ ).

Daily fluoxetine doses ranged from 10 to $40 \mathrm{mg}$ (one subject on $10 \mathrm{mg}$, one subject on $20 \mathrm{mg}$, two subjects on $40 \mathrm{mg}$ ). Duration of fluoxetine treatment ranged from three to 12 months (one subject for three months, one subject for four months, one subject for eight months, one subject for 12 months).

Blood samples for HPLC measurement of either serum fluvoxamine, or serum fluoxetine and norfluoxetine concentrations were taken within 1 hour prior to the MRS measurement. Estimations of steady state or washout concentrations were calculated from MRS recordings performed after a minimum of 30 days treatment for fluvoxamine and three months treatment for fluoxetine. These minimum treatment durations taken for establishment of steady state are compatible with reported brain uptake pharmacokinetic data for fluvoxamine (Strauss et al. 1997) and fluoxetine (Karson et al. 1993). Steady state assessments were performed from one to four times per subject during the steady state period, with the reported $n$ being the number of assessments. One subject had the MRS measurement without the corresponding blood sample for plasma assessment and another had a blood sample taken without having a corresponding MRS exam for brain assessment: data from these unpaired brain or plasma assessments at steady state for fluvoxamine treatment were included in the respective brain or plasma steady state average calculation, but not in the brain-to-plasma ratio or correlation calculations which require paired data points.

Repeated washout pharmacokinetic recordings were performed on four subjects after abrupt withdrawal from fluvoxamine, and on two subjects after abrupt withdrawal from fluoxetine. Abrupt withdrawal means that the subjects stopped taking the drug entirely on the first day of withdrawal. Time points for kinetic data are reported in hours after the last drug administration. None of the subjects reported any undesirable withdrawal effects.

\section{Plasma Concentrations by HPLC}

Plasma fluvoxamine or fluoxetine and norfluoxetine concentrations were measured from serum of blood samples which were taken within one hour of the of MRS recordings.

A high performance liquid chromatography (HPLC) method was developed in-house to separate and dose fluvoxamine in human serum. The method uses clovox- 
amine as an internal standard. An initial extraction of the serum in alkaline medium $(\mathrm{NaOH} 2 \mathrm{M})$, using a heptane-isopropanol mixture, is followed by a hydrochloric acid $(0.1 \mathrm{~N})$ extraction. An amount of $30 \mu \mathrm{l}$ of the aqueous phase is then injected on a Nucleosil C8 $5 \mu$ column $(150 \times 4.6 \mathrm{~mm})$. A phosphate buffer-acetonitril mixture is used for the mobile phase which has a flow of $1.1 \mathrm{ml} /$ minute. The absorption wavelength used is $190 \mathrm{~nm}$. The retention time is 4.7 minutes for the internal standard and 6.2 minutes for fluvoxamine.

A HPLC method was also developed in-house to separate and dose fluoxetine and norfluoxetine in human serum. The method uses desmethylclomipramine as an internal standard. An initial extraction of the serum in alkaline medium $(\mathrm{NaOH} 2 \mathrm{M})$, using a hexane-isoamylic alcohol mixture, is followed by a phosphoric acid $(0.05 \%)$ extraction. An amount of $30 \mu$ l of the aqueous phase is then injected on an Ultrasphere ODS $5 \mu$ column $(250 \times 4.6 \mathrm{~mm})$. A phosphate buffer-acetonitril mixture is used for the mobile phase which has a flow of $2.0 \mathrm{ml} /$ minute. The absorption wavelength used is 214 $\mathrm{nm}$. The retention time is 7 minutes for norfluoxetine, 9.1 minutes for fluoxetine, and 12.1 minutes for the internal standard. The calibration range is performed with an $\mathrm{O}$ serum loaded with fluoxetine and norfluoxetine with the final fluoxetine and norfluoxetine concentrations reaching 50,100, 250, and $500 \mu \mathrm{g} / \mathrm{l}$, respectively. Measurement accuracy was assessed during the dosing procedure in a concentration range from 0 to $50 \mu \mathrm{g} / 1$.

\section{Proton and Fluorine MR Recordings}

MR spectroscopy and imaging were performed on a Medspec 3 Tesla spectrometer (Bruker Spectrospin SA, Wissembourg, France) using UXNMR/Paravision acquisition software (Bruker Medizintechnik GMBH, Ettlingen, Germany). Separate proton and fluorine head coils were used for recordings in two steps after subject positioning. Both coils had identical geometry and design, i.e., they were linear cylindrical bird cage head coils with a $28-\mathrm{cm}$ inner diameter. For the duration of the recordings, the subject lay supine with head resting at the center of the magnet on a head support around which the RF coils were placed. First shimming and frequency adjustments were performed using the proton head coil tuned to $125 \mathrm{MHz}$. With the proton coil in place, pilot images in the three planes were recorded to verify adequate positioning, and a proton single pulseacquire spectrum was recorded to verify adequate shimming.

The shimming was considered acceptable if the line width at half-height of the water peak from the whole head was not larger than $25 \mathrm{~Hz}$. Proton 3-dimensional (3D) images with a matrix size of $128 \times 128 \times 128$, and a field of view (FOV) of $25.6 \mathrm{~cm}$ in each dimension were acquired for anatomical referencing of head spectro- scopic images. A gradient echo imaging sequence with a non-selective square excitation pulse preceded by a non-selective adiabatic inversion pulse, was used for the $3 \mathrm{D} \mathrm{T}_{1}$ weighted images. After removal of the proton coil from the back of the magnet without movement of the subject, the identically positioned linear fluorine head coil was used for fluorine spectroscopy at 117 MHz. A $10 \mathrm{ml}$ cylindrical sealed vial of a solution of 10 $\mathrm{mM}$ trifluoroethanol (TFE) in water attached to the head support was used as an external quantitative reference.

For estimations of brain concentration, fluorine pulse-acquire spectroscopy of the whole head was performed with a repetition time (TR) of 1s, a flip angle of $90^{\circ}$, a spectral width (SW) of $20000 \mathrm{~Hz}$, and data size of 16384 points. For localization of signal from the drug in vivo, two-dimensional fluorine MR spectroscopic images (MRSI) (Brown et al. 1982) of the head were performed at steady state before the washout period as described by Bolo et al. (1997): at the nominal fluorine resonance frequency $117 \mathrm{MHz}$ with RF transmitter frequency centered between the fluvoxamine and TFE resonances, in the axial $(x-y)$ plane with no slice selection, with a matrix size of $8 \times 8$ for 2 -D phase encoding. Since there was no slice selection, the MRSI images can be considered as peak area projections in the axial plane from the volume inside the head coil. Other parameters of the MRSI were: raw data free induction decay (FID) size $=2048$ points, $\mathrm{SW}=15151 \mathrm{~Hz}, \mathrm{TR}=1 \mathrm{~s}, \mathrm{FOV}=$ $25.6 \times 25.6 \mathrm{~cm}, \mathrm{NS}=16$ averages for a total experiment time of 17 minutes.

Spectral processing was performed with UXNMR/ Paravision software (Bruker Medizintechnik GMBH, Ettlingen, Germany) blindly to any information concerning subjects, treatments, doses, or plasma concentrations. Image processing for segmentation, volume measurements, and tissue density projections was done with software developed in-house. For the whole head non localized recordings used to estimate brain concentrations, resolution enhancement by zero filling was applied in order to qualitatively separate fluoxetine and norfluoxetine peaks in vivo without introducing artefacts, notwithstanding the experimental conditions which did not allow sufficient separation for quantitation purposes. Apodization with a matched filter was applied in order to increase the signal-to-noise ratio. The raw data FIDs from the whole head recordings were zero filled to 32768 points and were apodized with an exponential filter resulting in $50 \mathrm{~Hz}$ line broadening before Fourier transformation to spectra. In order to increase the accuracy of numerical integration, minor manual interactive spectral baseline corrections were performed on a small number of spectra for which the noise in the signal free portion was not evenly scattered around the zero line. Numerical integration of the TFE peak and the fluvoxamine peak or the combined fluox- 
etine-norfluoxetine (CF-norfluoxetine) peak was performed using 18 ppm-wide $(2106 \mathrm{~Hz})$ integration regions.

To obtain images of drug localization (Figure 1), the MRSI data were zero filled to a matrix size of $64 \times 64$ in the spatial dimensions which were apodized with a sine-squared filter in order to increase the spatial resolution of the images without introducing artefacts as was verified by inspecting images of noise regions. Before MRSI image formation the FIDs were apodized in the spectral dimension with an exponential filter resulting in $20 \mathrm{~Hz}$ line broadening in order to increase the signal-to-noise ratio. Spectroscopic images were obtained over $118 \mathrm{~Hz}$ wide spectral regions centered on the resonances of interest. To obtain localized spectra (Figure 2), the MRSI data were zero filled to 16384 points in the spectral dimension which was apodized with an exponential filter resulting in $50 \mathrm{~Hz}$ line broadening in order to increase the signal-to-noise ratio. Localized spectra were then obtained by Fourier transformation followed by a magnitude calculation. Tissue density images in the axial plane (Figures 1e-f) were obtained by operator interactive segmentation of the 3D $\mathrm{T}_{1}$ weighted images using in-house software; in these images the relative density of a given structure (e.g., brain tissue) at each pixel location is defined as the number of voxels of the segmented structure that project onto the pixel location in the axial plane divided by the total number of voxels in the 3D image set. Density of head tissue other than brain excluding cerebro spinal fluid (CSF) was obtained by density image subtraction. Spectroscopy and MRSI of the legs was also performed in this study in a manner similar to the head recordings, but results are not reported in this paper.

\section{MRS Estimations of Tissue Concentrations}

Spectra obtained from solutions in vitro of fluoxetine dissolved in physiological saline with concentrations ranging from 0.11 to $1.76 \mathrm{mM}$ demonstrated linearity between drug concentration and signal intensity for the hardware configuration used in this study $\left(\mathrm{r}^{2}=1.00\right)$. Estimations of the amount of brain fluvoxamine or CFnorfluoxetine in micromoles were obtained by taking the ratio of the drug peak integral to the TFE peak integral times the amount of TFE in the external reference and correcting for $\mathrm{T}_{1}$ decay effects. Thus, the absolute concentration $\mathrm{C}$ in $\mu \mathrm{M}$ was estimated using the following formula:

$$
C=\frac{I_{d r u g}}{I_{T F E}} \times \frac{A_{T F E}}{V_{\text {brain }}} \times \operatorname{Corr}_{T_{1}}
$$

with $\mathrm{I}_{\text {drug }}$ and $\mathrm{I}_{\mathrm{TFE}}$ being, respectively, the drug and TFE integrals, $A_{\text {TFE }}$ the amount of TFE in the external reference $(100 \mu \mathrm{m})$, and $\mathrm{V}_{\mathrm{brain}}$ the brain volume. Corr $\mathrm{T}_{\mathrm{T} 1}$ cor- rects for differences in saturation between the brain and the external reference and is given by the following for $90^{\circ}$ pulses:

$$
\operatorname{Corr}_{T_{1}}=\frac{\left(1-\exp \left(-T R / T_{1 T F E}\right)\right)}{\left(1-\exp \left(-T R / T_{1} \text { drug }\right)\right)}
$$

with $T_{1}$ TFE and $T_{1}$ drug being, respectively, the TFE and drug $T_{1}$ values. In this study we have not attempted to measure the relaxation times in vivo of fluvoxamine or fluoxetine. The $\mathrm{T}_{1}$ of fluvoxamine ${ }^{19} \mathrm{~F}$ in brain tissue at a field strength of $1.5 \mathrm{~T}$ has been estimated in a group of subjects with obsessive compulsive disorder to be in the range of ca. 140 to $230 \mathrm{msec}$ (Strauss et al. 1997). The $\mathrm{T}_{1}$ of fluoxetine ${ }^{19} \mathrm{~F}$ in brain tissue at a field strength of $1.5 \mathrm{~T}$ has been estimated to be ca. $190 \mathrm{~ms}$ (Karson et al. 1993). We assume that the $T_{1}$ 's are in a similar range in this study, and have taken an approximate intermediate value of $180 \mathrm{~ms}$ for the drug $\mathrm{T}_{1}$ in vivo to calculate the $\mathrm{T}_{1}$ correction term. The $T_{1}$ of the TFE external reference was measured with an inversion recovery $180^{\circ}-\mathrm{TI}-90^{\circ}$ sequence, with an adiabatic $180^{\circ}$ inversion pulse followed by a spoiler gradient pulse to destroy residual transverse components during the inversion delay TI.

The effect of relaxation during RF pulse and receiver dead-time $\left(\mathrm{T}_{\mathrm{d}}\right)$ on concentration measurement error was assessed and was found to be negligible in our case. The $90^{\circ}{ }^{19} \mathrm{~F}$ RF pulses were $100 \mu$ s long and thus short enough to neglect the $\mathrm{T}_{1}$-like relaxation occuring during the pulse. The dead-time was $250 \mu$ s long. The $\mathrm{T}_{2}{ }^{* \prime} \mathrm{s}$ of fluvoxamine, combined fluoxetine-norfluoxetine and the external reference were estimated by spectral fitting for a sample of data points and found to be 9 $\pm 3 \mathrm{~ms}, 9 \pm 3 \mathrm{~ms}$, and $10 \pm 2 \mathrm{~ms}$, respectively. The $\mathrm{T}_{2}{ }^{*}$ correction factor:

$$
\operatorname{Corr}_{T_{2}^{*}}=\frac{\exp \left(-T_{d} / T_{2 T F E}^{*}\right)}{\exp \left(-T_{d} / T_{2 d r u g}^{*}\right)}
$$

thus yielded a value ranging from 1.003 to 1.02 and was neglected for all concentration calculations.

The fluvoxamine signal originates from all fluvoxamine inside the volume of the fluorine RF coil. To calculate the brain concentration, it is assumed to originate primarily from the brain, which is consistent with the ${ }^{19} \mathrm{~F}$ MRSI results obtained in this study. Brain volumes for each subject were estimated from the 3D proton images after segmentation of brain tissue (excluding CSF). Since the external reference was present next to the head in each experiment, variations in signal amplitude with coil loading were considered to affect both the external reference and tissue drug signals identically therefore eliminating the need to make corrections for coil loading. Since the fluorine MRS peaks of the two drugs and the external reference arise from a $\mathrm{CF}_{3}$ group, no correction for number of fluorine nuclei per molecule ( $n=3$ for the $\mathrm{CF}_{3}$ group) was needed either. 

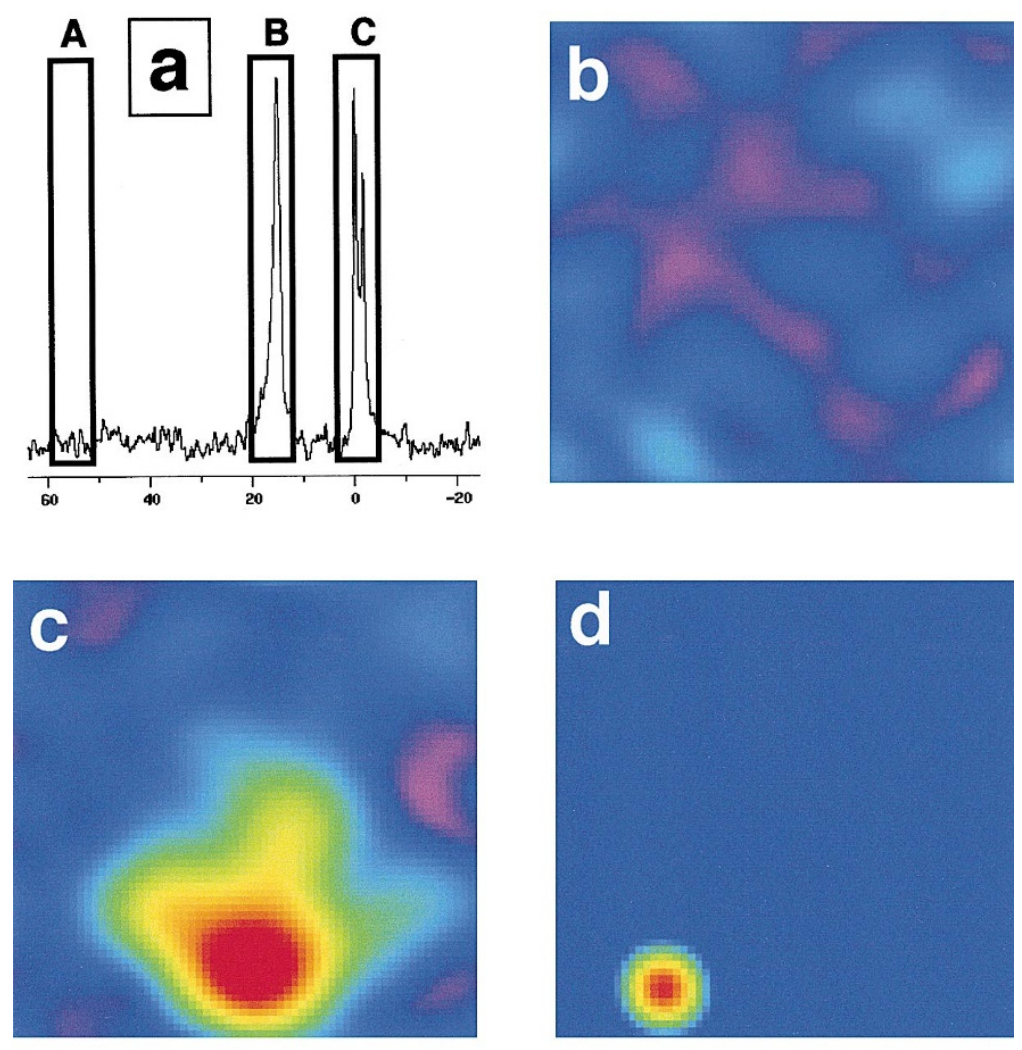

Figure 1. Spectroscopic imaging and tissue density images of the head of fluvoxamine treated subject $\mathrm{km}$. (a) Spectral regions used for ${ }^{19} \mathrm{~F}$ MRSI images: boxes are delimited on a spectrum in vivo of the whole head with the external reference present. $\mathrm{A}=$ noise region (for Figure $1 b), B=$ fluvoxamine region (for Figure 1c), $C=$ trifluoroethanol (TFE) external reference region (for Figure 1d). (b) MRSI of noise region. (c) MRSI of fluvoxamine region. (d) MRSI of TFE region. (e) distribution of brain tissue density in the axial plane (identical to MRSI plane). (f) distribution of head tissues other than brain in the axial plane (identical to MRSI plane).
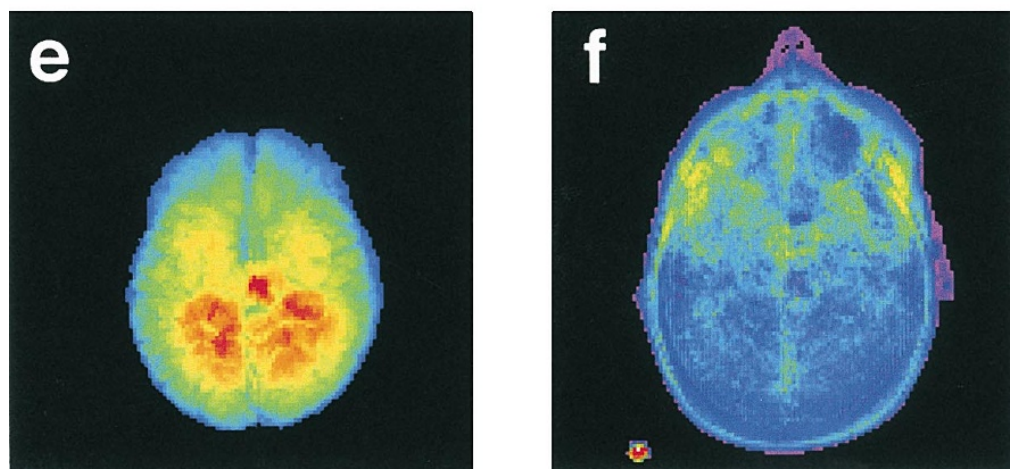

Individual ratios of brain to plasma concentrations at steady state were calculated for each measurement on each subject and the means and standard deviations of these ratios are reported. The error on the concentration value was estimated for each measurement from the calculated relative errors on the values of the integrals (drug and external reference) due to signal to noise according to Weiss and Ferretti (1983). For the entire fluvoxamine study the relative error on the concentration value was on average $2.9 \%$ and ranged from $0.9 \%$ to $8.6 \%$ for 121 measurements. For the entire fluoxetine study the relative error on the concentration value was on average $2.9 \%$ and ranged from $1.0 \%$ to $6.5 \%$ for 39 measurements. The paired t-test $\mathrm{r}^{2}$ and the Pearson correlation coefficient were used to statistically evaluate differences and correlation between brain and plasma concentrations and also correlation between age and plasma, brain concentrations or brain-to-plasma ratios.

\section{Analysis of Washout Data}

For ${ }^{19} \mathrm{~F}$ signal from the head, analysis of washout data was performed on the estimated brain concentrations. No correction was made for signal arising from circulating blood which constitutes approximately $3 \%$ of brain volume in vivo. Experimental washout data points of estimated drug concentration vs. time were analyzed by fitting a three parameter monoexponential decay curve:

$$
\mathrm{C}(\mathrm{t})=\mathrm{A} \cdot \exp (-\mathrm{K} \cdot \mathrm{t})+\mathrm{B}
$$

with $\mathrm{A}=$ span in $\mu \mathrm{M}, \mathrm{K}=$ rate constant in (hours) ${ }^{-1}, \mathrm{t}=$ time in hours, and $\mathrm{B}=$ plateau in $\mu \mathrm{M}$, to the data points 


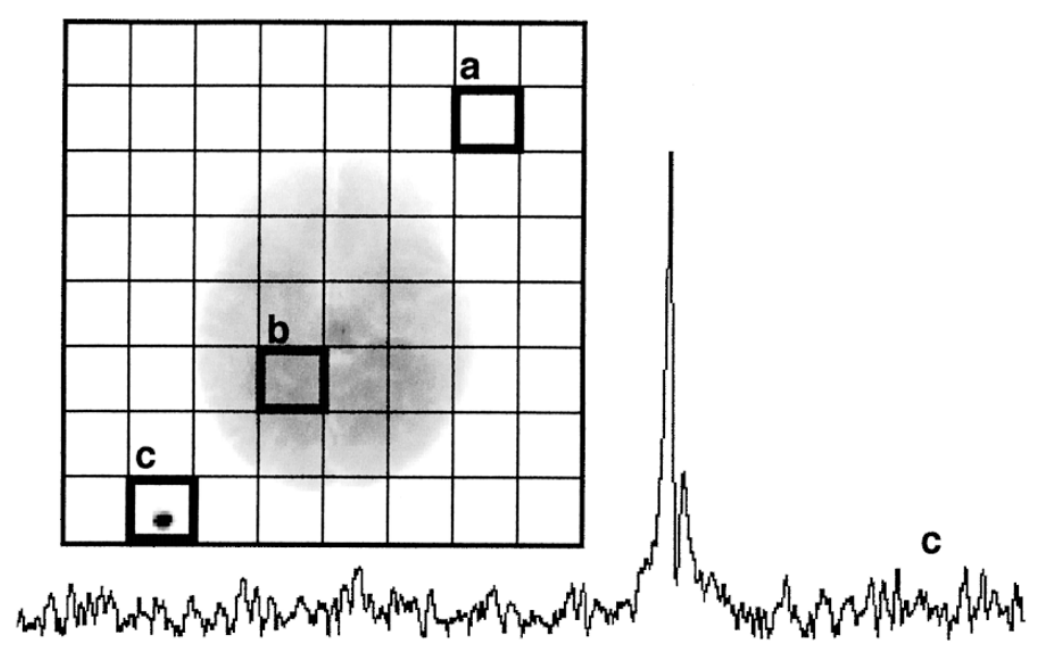

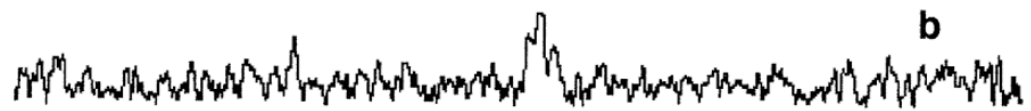

Figure 2. Localized ${ }^{19} \mathrm{~F}$ spectra from the MRSI (subject $\mathrm{km}$ ). Localizations of the selected ${ }^{19} \mathrm{~F}$ magnitude spectra are spatially referenced by overlaying the MRSI voxel grid on images of the relative densities of brain and external reference in the axial plane. (a) ${ }^{19} \mathrm{~F}$ spectrum from voxel not containing any structure. (b) ${ }^{19} \mathrm{~F}$ spectrum from voxel containing brain tissue. (c) ${ }^{19} \mathrm{~F}$ spectrum from voxel containing the external reference vial.

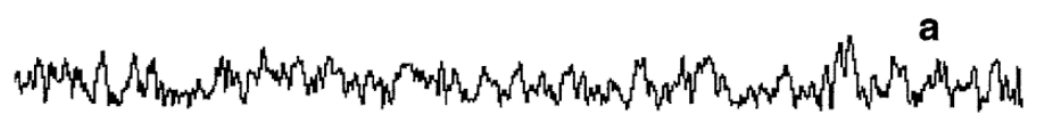

by a non-linear regression method minimizing sum-ofsquares (GraphPad-Prism 1995). Plasma concentration decays were analyzed in the same manner by ommiting the plateau B. Bi-exponential decay curves fit the data more poorly than the mono-exponential ones and were therefore not considered for the analysis. Half-lives reported are $0.69 / \mathrm{K}$. Values of estimated curve parameters are reported with the curve goodness-of-fit parameter $r^{2}$. The validity of the model was verified with the test of runs in each case. The ratios of kinetic parameters (half-life and decay constant K) were calculated for each subject and the means and standard deviations of these ratios are reported.

\section{RESULTS}

\section{MRSI Results}

Figure 1 (a-f) shows typical ${ }^{19} \mathrm{~F}$ MR spectroscopic images recorded in the axial plane without slice selection of the head of a fluvoxamine treated subject compared to proton $3 \mathrm{D} \mathrm{T}_{1}$ weighted imaging data recorded in the same session. The subject $\mathrm{km}$ whose data is shown had been taking $300 \mathrm{mg}$ a day of fluvoxamine for one year prior to the MR recordings. Her serum fluvoxamine concentration measured by HPLC within an hour of the MRSI exam was $0.72 \mu \mathrm{M}$, and the estimated brain concentration was $8.5 \mu \mathrm{M}$. The spectral regions which were used for the MRSIs are shown in Figure 1a on a spectrum in vivo of the whole head with the external reference present. Head MRSIs of the spectral regions of noise (spectral region $A$ in Figure 1a), fluvoxamine (spectral region B in Figure 1a), and TFE (spectral region $C$ in Figure 1a) recorded at steady state are shown in Figures 1b, c, and d, respectively. Figure 1e shows brain tissue density whereas Figure 1f shows the density of head tissue other than brain (excluding CSF and bone) plus the density of the external TFE reference. All densities are shown in the axial plane. Comparison of the head fluvoxamine MRSI (Figure 1c) to the brain density (Figure 1e) demonstrates a similarity of intensity distribution. Conversely, there is a poor correspondence between the fluvoxamine MRSI and the densities of other tissues (skin and muscle) excluding brain (Figure 1f). The external reference MRSI intensity distribution corresponds to its position as is seen by comparing Figures $1 \mathrm{~d}$ and $\mathrm{f}$. The MRSI of the noise spectral region (Figure 1b) showed no high intensity areas, indicating that the MRSIs were free of artefacts.

Localized magnitude spectra selected from the MRSI data set are shown in Figure 2. The MRSI axial plane spatial grid in Figure 2 is overlayed on the sum of brain and external reference vial relative density images in order to spatially reference the localization of the spectra relative to these structures. In the MRSI grid each voxel for spectral localization has a volume of approximately $260 \mathrm{ml}$. Sample spectra shown were selected from voxels localized outside of either head or external reference $(2 a)$, through the head $(2 b)$, and containing the external reference (2c). The spectrum from the voxel not containing any structure shows only noise, which is consistent with an artefact free MRSI data set. The spectrum from the voxel localized in the head shows a single peak at the fluvoxamine frequency. The spectrum 
Table 1. Steady-State Brain and Plasma Estimated Concentrations

\begin{tabular}{lcrcrcr}
\hline & $\begin{array}{c}\text { Brain } \\
\text { Conc. } \\
(\boldsymbol{\mu M})\end{array}$ & \multicolumn{1}{c}{$\boldsymbol{n}$} & $\begin{array}{c}\text { Plasma } \\
\text { Conc. } \\
(\boldsymbol{\mu M})\end{array}$ & \multicolumn{1}{c}{$\begin{array}{c}\text { Brain/Plasma } \\
\text { Ratio }\end{array}$} & $\boldsymbol{n}$ \\
\hline Frug & $12 \pm 5$ & 13 & $1.12 \pm 0.31$ & 13 & $10 \pm 2$ & 12 \\
Fluvoxamine $^{*}$ & $13 \pm 7$ & 4 & $1.73 \pm 1.00$ & 4 & $10 \pm 6$ & 4 \\
\hline
\end{tabular}

Average values are reported in $\mu \mathrm{M}$ ( \pm standard deviation) of fluvoxamine or combined fluoxetine-norfluoxetine with brain-to-plasma ratios $\mathrm{n}=$ number of measurements. Fluoxetine ${ }^{*}=$ fluoxetine + norfluoxetine

from the voxel containing the external reference shows a single peak at the TFE frequency.

\section{Steady State Brain and Plasma Concentrations}

The steady state concentrations and brain/plasma ratios of fluvoxamine and combined fluoxetine-norfluoxetine are similar as is shown in Table 1. For both drugs, the brain and plasma concentrations were significantly correlated as is shown in Figure 3, in which brain concentrations are plotted vs. plasma concentrations.

For fluvoxamine, the estimated brain tissue concentration at steady state for these subjects was $12 \pm 5 \mu \mathrm{M}$ (mean $\pm \mathrm{SDV}, n=13$ ) and ranged from 6 to $24 \mu \mathrm{M}$. The steady state plasma concentration was $1.12 \pm 0.31 \mu \mathrm{M}$ (mean $\pm \mathrm{SDV}, n=13$ ) and ranged from 0.71 to $1.75 \mu \mathrm{M}$. Brain concentrations were significantly higher than plasma concentrations $(p=.000005)$. The brain to plasma concentration ratio was $10 \pm 2$ (mean \pm SDV, $n=12$ ) and ranged from 6 to 14 . Furthermore, brain fluvoxamine concentrations were positively correlated with plasma concentrations $\left(\mathrm{r}^{2}=0.69, n=12\right)$ (Figure $3)$. No significant difference between men and women was found for either brain or plasma concentrations. No correlation was found between brain concentrations and either treatement dose, treatment duration, or cumulated dose (product of the daily dose with the duration of treatment in days). No correlation was found between age and either plasma concentrations, brain concentrations, or brain-to-plasma ratios.

Since the MR resonances of fluoxetine and its active metabolite norfluoxetine cannot be discriminated in the experimental conditions of this study, brain concentrations were estimated for their combination and were compared to the combined plasma concentrations. The estimated brain tissue concentration of combined fluoxetine-norfluoxetine (CF-norfluoxetine) at steady state for these subjects was $13 \pm 6 \mu \mathrm{M}$ (mean $\pm \mathrm{SDV}, n=4$ ) and ranged from 5 to $17 \mu \mathrm{M}$. The steady state plasma

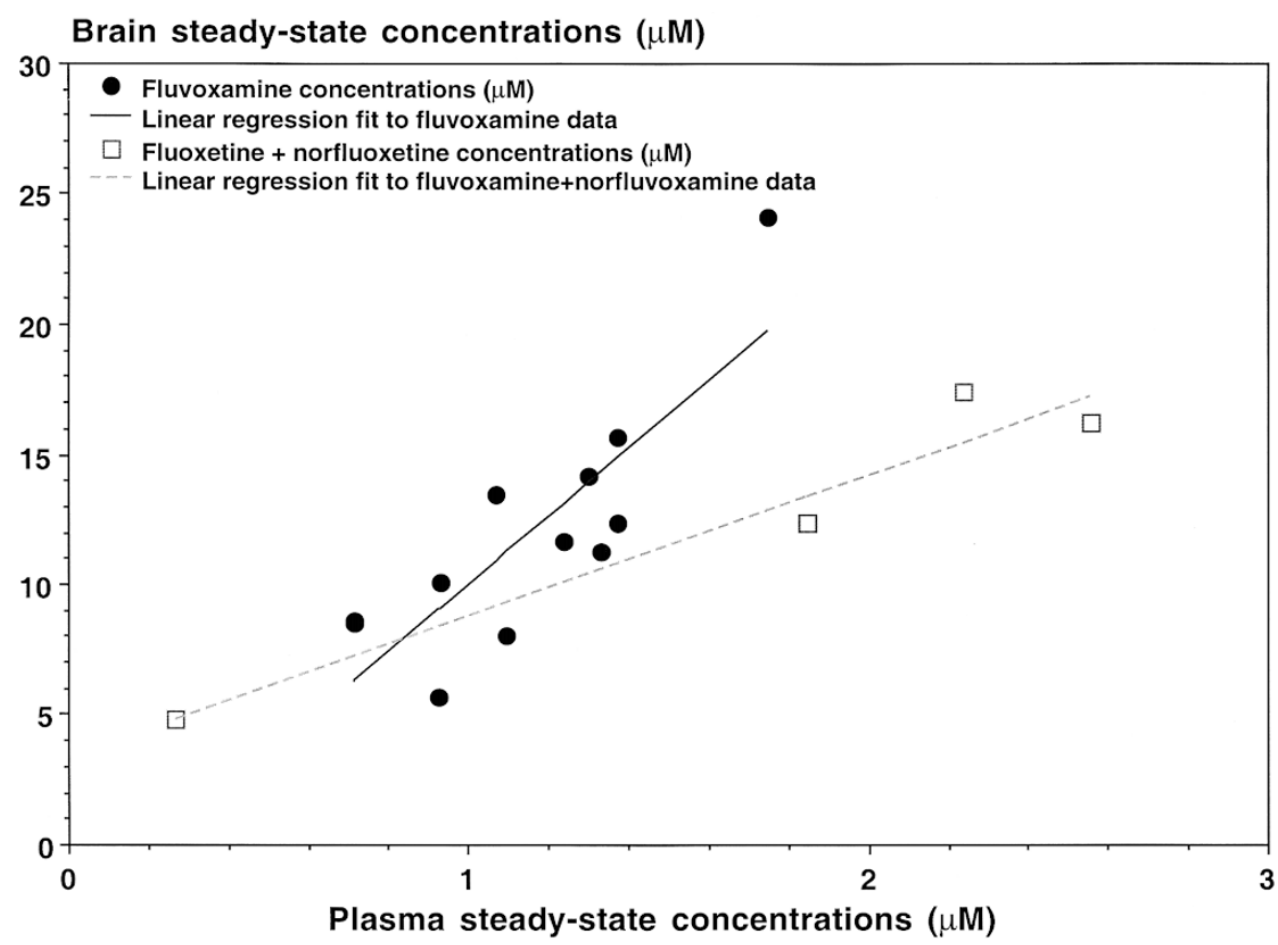

Figure 3. Correlation of steady state brain concentrations to plasma concentrations for fluvoxamine and combined fluoxetine-norfluoxetine. Filled circles represent fluvoxamine concentration data points and the full line represents the linear regression fit to fluvoxamine data. Empty squares represent combined fluoxetine-norfluoxetine concentration data points and the broken line represents the linear regression fit to combined fluoxetine-norfluoxetine data. 
concentration was $1.73 \pm 1.00 \mu \mathrm{M}($ mean $\pm \mathrm{SDV}, n=4)$ and ranged from 0.3 to $2.6 \mu \mathrm{M}$.

Brain concentrations were significantly higher than plasma concentrations $(p=.004)$. The brain to plasma concentration ratio was $10 \pm 6$ (mean \pm SDV,$n=4)$ and ranged from 6 to 18. Furthermore brain CF-norfluoxetine concentrations were positively correlated with plasma concentrations $\left(\mathrm{r}^{2}=0.94, n=4\right)$ (Figure 3). Since steady state values for CF-norfluoxetine brain and plasma were obtained on a smaller population sample size (three women and one man) than for fluvoxamine a statistical comparison between men and women was not performed and other correlations tested should be interpreted with caution. No significant correlation was found between brain concentrations and either treatment dose, treatment duration, or cumulated dose (product of the daily dose with the duration of treatment in days). Positive correlations were found between age and plasma concentrations $\left(\mathrm{r}^{2}=0.86, n=4\right)$, brain concentrations $\left(\mathrm{r}^{2}=0.92, n=4\right)$, and brain-toplasma ratios $\left(\mathrm{r}^{2}=0.93, n=4\right)$.

\section{Washout After Abrupt Discontinuation of Treatment}

The results of the analysis of the fluvoxamine and CFnorfluoxetine washout pharmacokinetics in brain tissue and plasma are summarized in Table 2 which presents initial concentrations $\left(\mathrm{C}_{0}\right)$, plateaus $(\mathrm{B})$, half-lives, and brain-to-plasma half-life ratios for each subject. For all of the fits, the test of runs did not detect any deviation from the model of monoexponential decay. Figure 4 presents typical results of estimated brain and plasma concentrations and the exponential decay fits to the data during fluvoxamine washout after abrupt discontinuation for one subject. The brain tissue fluvoxamine washout half-life of $79 \pm 24$ hours $(n=4)$ was found to be $2.2 \pm 0.3$ times longer $(n=4)$ than its plasma half-life of $35 \pm 8$ hours $(n=4)$. The brain tissue fluoxetine halflife of $382 \pm 48$ hours $(n=2)$ was found to be close its plasma half-life of $406 \pm 172$ hours (ratio of half-lives $=$ $1.0 \pm 0.3$ ) with only two subjects being studied for their tissue kinetics. Fluvoxamine brain and plasma halflives were significantly shorter than those of fluoxetine.

\section{DISCUSSION}

In this preliminary study we were able to compare the steady state and pharmacokinetic values of fluvoxamine and combined fluoxetine-norfluoxetine in brain in vivo using ${ }^{19} \mathrm{~F}$ MRS, and in plasma using HPLC in a group of subjects under treatment for a major depressive episode. Quantitative estimations by ${ }^{19} \mathrm{~F}$ MRS were obtained using an external reference and by integrating over broad spectral regions thus taking into account variations of the experimental conditions of coil loading and magnetic field inhomogeneities. Localization of the tissue from which the drug signal in vivo primarily originates was also possible from MRSI recordings performed in the head.

\section{Drug Localization and Distribution}

In agreement with localization studies of fluoxetine (Komoroski et al. 1994), we found that fluvoxamine sig-

Table 2. Brain and Plasma Pharmacokinetic Parameters from Washout Results

\begin{tabular}{|c|c|c|c|c|c|c|c|c|c|c|}
\hline \multirow[b]{2}{*}{ Subject } & \multicolumn{5}{|c|}{ Results from Brain Data } & \multicolumn{3}{|c|}{ Results from Plasma Data } & \multicolumn{2}{|c|}{ Brain/Plasma Ratios } \\
\hline & $\begin{array}{l}\text { Initial Conc. } \\
C(0)(\mu M)\end{array}$ & $\begin{array}{l}\text { Plateau } \\
\text { B }(\mu \mathrm{M})\end{array}$ & $\begin{array}{l}\text { Half-life } \\
\text { (Hours) }\end{array}$ & $\mathbf{r}^{2}$ & $P($ runs) & $\begin{array}{c}\text { Span } \\
\mathbf{A}(\mu \mathrm{M})\end{array}$ & $\begin{array}{l}\text { Half-Life } \\
\text { (Hours) }\end{array}$ & $\mathbf{r}^{2}$ & $\begin{array}{c}\text { B/P Ratio } \\
\text { Half-Lives }\end{array}$ & $\begin{array}{c}\text { B/P Ratio } \\
\text { C(0) }\end{array}$ \\
\hline \multicolumn{11}{|l|}{ FLUVOXAMINE } \\
\hline $\mathrm{dg}$ & $15 \pm 4$ & $2 \pm 2$ & 77 & 0.92 & 0.84 & $1.32 \pm 0.02$ & 40 & 0.99 & 1.94 & $\begin{array}{r}11.13 \\
980\end{array}$ \\
\hline vn & $12 \pm 3$ & $4 \pm 1$ & 49 & 0.89 & 0.70 & $1.24 \pm 0.01$ & 25 & 0.99 & 1.97 & $\begin{array}{r}9.80 \\
1139\end{array}$ \\
\hline $\mathrm{km}$ & $16 \pm 1$ & $0 \pm 1$ & 107 & 0.99 & 0.71 & $1.37 \pm 0.02$ & 44 & 0.99 & 2.44 & 11.39 \\
\hline lc & $9 \pm 1$ & $2 \pm 1$ & 83 & 0.99 & 0.80 & $0.42 \pm 0.05$ & 32 & 0.98 & 2.56 & 20.68 \\
\hline Mean & 13 & 2 & 79 & & & 1.09 & 35 & & 2.23 & 13.25 \\
\hline SDV & 3 & 2 & 24 & & & 0.45 & 8 & & 0.32 & 5.00 \\
\hline \multicolumn{11}{|l|}{$\begin{array}{l}\text { FLUOXETINE- } \\
\text { NORFLUOXETINE }\end{array}$} \\
\hline dj & $16 \pm 4$ & $0 \pm 3$ & 349 & 0.92 & 0.19 & $2.52 \pm 0.09$ & 284 & 0.98 & 1.23 & 6.36 \\
\hline $\mathrm{lb}$ & $18 \pm 1$ & $0 \pm 3$ & 416 & 0.99 & 0.50 & $2.28 \pm 0.19$ & 528 & 0.89 & 0.79 & 7.90 \\
\hline Mean & 17 & 0 & 382 & & & 2.40 & 406 & & 1.01 & 7.13 \\
\hline SDV & 2 & 0 & 48 & & & 0.17 & 172 & & 0.31 & 1.09 \\
\hline
\end{tabular}

Fluvoxamine and fluoxetine-norfluoxetine washout non-linear regression fitted curve parameters. The exponential decay function $\mathrm{C}(\mathrm{t})=\mathrm{A}$ $\exp (-\mathrm{K} / \mathrm{t})+\mathrm{B}$ was fitted to the estimated tissue concentration curves, with $\mathrm{A}$ and $\mathrm{B}$, respectively, the span and plateau of the decay. The initial concentration $C(0)$ is the sum $A+B$, the half life is $0.69 / K, r^{2}$ is the goodness of fit, and $P($ runs) is the P value for the runs test. Parameters $K$, A and B are presented with their standard error $( \pm \mathrm{SE}) . \mathrm{SDV}=$ standard deviation. 
Tissue fluvoxamine concentrations $(\mu \mathrm{M})$

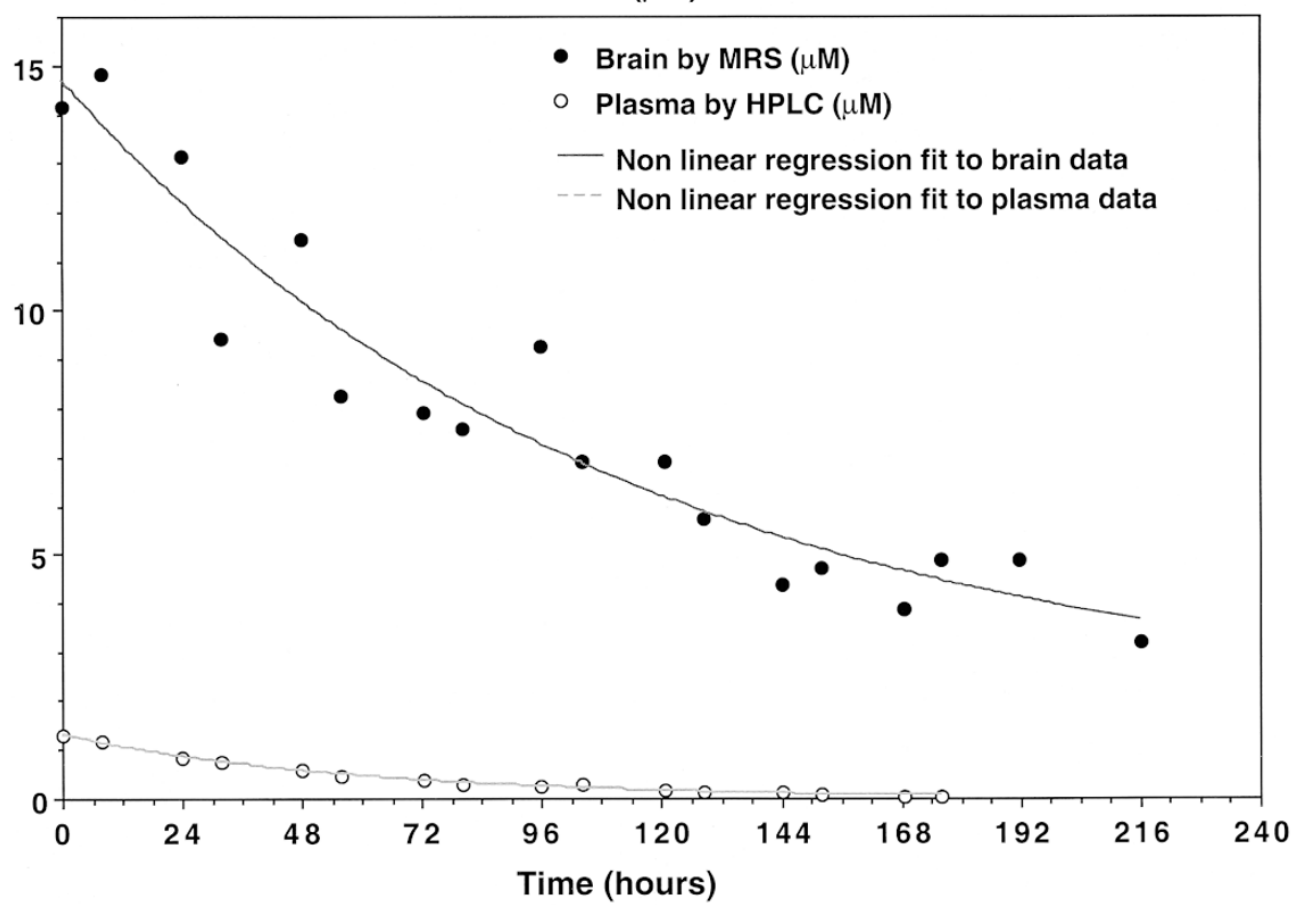

Figure 4. Fluvoxamine tissue pharmacokinetics for subject dg. Filled and empty circles represent the estimated concentrations in $\mu \mathrm{M}$ for brain tissue (estimated by ${ }^{19} \mathrm{~F}$ MRS) and plasma (measured by HPLC), respectively. The solid and dotted lines represent the respective exponential decay curves fitted to the data.

nal recorded in vivo from the whole head originates primarily from brain tissue. Comparison of the drug signal intensity distribution in the MRSI to the brain density in the same imaging plane furthurmore suggests that the ${ }^{19} \mathrm{~F}$ MRS visible drug, which is the unbound form, is homogeneously distributed in the brain. This homogeneous distribution does not preclude a heterogeneous distribution on specific binding sites, since specific binding represents at the therapeutic dose a negligible fraction of the total drug concentration detected by MRS.

Our finding that the steady state brain SSRI concentrations were about 10 times higher than plasma concentrations is compatible with its pharmacokinetic profile, with a reported apparent distribution volume of approximately 20 in humans for fluvoxamine (Palmer and Benfield 1994; Wilde et al. 1993) and 12 to 43 for fluoxetine (Altamura et al. 1994), indicating considerable uptake of the drug into tissue spaces. The result that no correlation was found between the steady state brain or plasma concentrations and the daily dose indicates that there can be a considerable variability of plasmatic levels relative to treatment dose. Furthermore, the lack of correlation of brain or plasma concentrations with either treatment duration or cumulated dose indicates that once steady state is attained for a subject further treatment does not affect the established state of bal- ance. These results confirm the low reliability of only taking into account drug dosage to appreciate the real impact of the drug on the brain. For the clinician, if the treatment is failing, the question is whether to increase the drug dosage or to change the treatment altogether. The good correlation found between brain and plasma concentrations comforts the view that the plasma level is a better indicator than dosage to help the clinician for the therapeutic decision.

\section{Estimation of Steady State Concentrations and Pharmacokinetic Parameters}

One goal of importance for this type of study is for the ${ }^{19} \mathrm{~F}$ MRS measurements to attain general clinical usefulness as an aid in explaining treatment and side effects. For this to occur, results between different studies using different methodologies should be reproducible. The steady state concentrations of fluvoxamine in brain tissue obtained in this study are in the same range as those found in other studies of the drug by ${ }^{19} \mathrm{~F}$ MRS notwithstanding differences in the methodology used for calibrating the estimated concentrations, i.e., external reference present during acquisition (this study) vs. precalibration with similarly loaded external reference (Strauss et al. 1997). Likewise, the fluvoxamine brain half-lives and ratios of brain to plasma half-lives were 
also in the same range. This suggests that if the calibration procedures take into account the factors affecting signal intensity (e.g., $\mathrm{T}_{1} \mathrm{~s}$ and coil loading) in order to obtain absolute concentrations then reproducibility of results between laboratories can be attained. Interestingly enough, the average steady state brain to plasma ratio was two times lower than that found in Strauss's (1997) study not because brain concentrations were lower but because plasma concentrations were about two times higher. This could be explained by differences in the subject populations: the subjects in this study were being treated for a major depressive episode, as opposed to obsessive compulsive disorder (OCD).

The steady state brain concentrations found for combined fluoxetine-norfluoxetine were also in the range of results found in the literature for ${ }^{19} \mathrm{~F}$ MRS studies (Renshaw et al. 1992; Miner et al. 1995) which also use calibration methods (precalibration with an external loading reference) different from this study. To our knowledge, washout kinetics of brain fluoxetine and norfluoxetine have not been studied by ${ }^{19} \mathrm{~F}$ MRS, but the values we found are compatible with uptake kinetics data (Renshaw et al. 1992). Since this study uses the same methodology to compare kinetics of the two SSRIs, and fluvoxamine results were consistent with those of other studies, it is suggested that fluoxetine results should be reproducible as well. An obvious shortcoming of the kinetic data is that the reported values result from the combination of fluoxetine and norfluoxetine data which are not separable under these conditions. As the two compounds have different plasma half-lives: 24 to 96 hours for fluoxetine vs. 168 to 360 hours for norfluoxetine, respectively (Altamura et al. 1994), their brain half-lives could as well present such a large discrepancy. A relatively large variability was found between the two subjects for which the brain fluoxetine-norfluoxetine half-life ranged from 349 to 416 hours. Brain half-lives were comparable to plasma half lives which demonstrated an even larger variability (284 and 528 hours, respectively). The kinetic results should be interpreted with caution, and considering the particularly small number of subjects studied under washout conditions in each treatment group, no definitive conclusion should be drawn for the brain pharmacokinetics.

Other important factors such as subject age and duration of treatment should be taken into account to compare the results of this study to others. However this study is limited by the the small population size and caution should be applied in the interpretation of its results. In this study, the age range as well as the treatment duration range were large with several subjects under treatment for about a year. No correlation was found between age and the steady state plasma or brain concentrations for the eight subjects treated with fluvoxamine whereas one was found for the four sub- jects under fluoxetine. If this preliminary result is confirmed with further studies, this would suggest different age related changes in metabolism for these two drugs. It appears of particular interest to continue this type of investigation of these SSRIs under long term treatment conditions.

As is with other studies, our data suffer from some of the usual shortcomings of ${ }^{19} \mathrm{~F}$ MRS investigations of this type in vivo. The inability to distinguish in vivo the SSRIs from their metabolites is related to chemical shift differences which are too small relative to the linewidth in vivo. This was the case for fluoxetine and norfluoxetine which, although visibly separated in the spectrum were not sufficiently so for quantitation purposes. Our measurements were made at a field strength of 3 Tesla for which ${ }^{19} \mathrm{~F} \mathrm{~T}_{1}$ values could be different than those measured at 1.5 Tesla. Since we did not measure $\mathrm{T}_{1}$ values, we cannot know if the the similarity of our results to those of other ${ }^{19} \mathrm{~F}$ MRS studies is related to the fact that we used the brain $T_{1}$ values from those studies to which we are comparing. Further investigation of ${ }^{19} \mathrm{~F} \mathrm{~T}_{1}$ values in vivo at high fields is still needed. Some of the dispersion in estimated concentration values could be due to the fact that for head measurements, since shimming optimizes field homogeneity preferentially on the head rather than on the external reference, the TFE signal was often distributed on a relatively wide frequency range. However, the large integral ranges used in this study were considered to take this into account.

\section{Subject Population and Clinical Usefulness}

In this study, plasma steady state concentrations obtained were found to be somewhat higher than the values from the literature for fluvoxamine (Palmer and Benfield 1994; De Vries et al. 1993) and fluoxetine (Altamura et al. 1994) studies. The discrepancies can be accounted for by the difference between single dose and long term multiple dose studies (Kelly et al. 1989) for which the values are comparable. It is notable that a relatively large variability has been found in the plasma parameters and some ${ }^{19} \mathrm{~F}$ MRS studies show distinctive outliers for brain concentrations or kinetic parameters.

In this study, the selection of subjects could have affected these parameters if a particular subset of the general population has been selected. The subjects in this study were all under treatment for a major depressive episode with the majority $(11 / 12)$ clearly diagnosed with major depressive disorder, whereas most data from the literature has been from subjects diagnosed with OCD. This could explain the differences found between our study and others. If confirmed by further investigations, these preliminary results would suggest that for major depressive disorder treated with these two SSRIs, plasma concentrations are higher than but brain concentrations are similar to those found in OCD, 
thus pointing to different metabolic profiles for these disorders.

\section{CONCLUSION}

The ability to track the individual pharmacodynamic parameters of target tissues as shown by this study leads us to believe that the ${ }^{19} \mathrm{~F}$ MRS investigative technique presents a great promise for providing both the clinician and the pharmaceutical industry with objective markers relevant to understanding drug therapeutic and side effects.

\section{REFERENCES}

Altamura AC, Moro A, Percudani M (1994): Clinical pharmacokinetics of fluoxetine. Drug Dispo 26(3):201-214

Bolo NR, Hodé Y, Macher JP (1996): Comparison of plasma and in-vivo brain levels of fluvoxamine measured by fluorine NMR spectroscopy during drug washout. In Proceedings of the International Society for Magnetic Resonance in Medicine; New York, NY, 2:1179

Bolo NR, Hodé Y, Hennel F, Nédélec JJ, Lainey E, Macher JP (1997): Localization of fluvoxamine signal in-vivo by fluorine chemical shift imaging at 3T. In Proceedings of the International Society for Magnetic Resonance in Medicine. Vancouver, Canada, 2:1431

Brown TR, Kincaid BM, Ugurbil K (1982): NMR chemical shift imaging in three dimensions. Proc Natl Acad Sci USA. 79:3523-3526

De Vries MH, Van Harten J, Van Bemmel P, Raghoebar M (1993): Pharmacokinetics of fluvoxamine maleate after increasing single oral doses in healthy subjects. Biopharm Drug Disp 14:291-296

Durst P, Schuff N, Crocq MA, Mokrani MC, Macher JP (1990): Non-invasive in-vivo detection of a fluorinated neuroleptic in the human brain by ${ }^{19} \mathrm{~F}$ nuclear magnetic resonance spectroscopy. Psychiatry Res Neuroimag 35:107-114

GraphPad-Prism (1995): Graph Pad Software Inc. Version 2.00
Karson CN, Newton JEO, Mohanakrishnan P, Sprigg J, Komoroski RA (1992): Fluoxetine and trifluoperazine in human brain: $\mathrm{A}^{19} \mathrm{~F}$-nuclear magnetic resonance spectroscopy study. Psychiatry Res Neuroimag 45(2):95-104

Karson CN, Newton JEO, Livingston R, Jolly JB, Cooper TB, Komoroski RA (1993): Human brain fluoxetine concentrations. J Neuropsychiatry Clin Neurosci 5:322-329

Kelly MJ, Perry PJ, Holstad SG, Garvey MJ (1989): Serum fluoxetine and norfluoxetine concentrations and antidepressant response. Therap Drug Monit 11:165-170

Komoroski RA, Newton JEO, Cardwell D, Sprigg J, Pearce J, Karson CN (1994): In vivo ${ }^{19} \mathrm{~F}$ spin relaxation and localized spectroscopy of fluoxetine in human brain. Magn Res Med 31:204-211

Komoroski RA, Newton JEO, Karson C, Cardwell D, Sprigg J (1990): Detection of psychoactive drugs in vivo in humans using ${ }^{19} \mathrm{~F}$ NMR spectroscopy. Biol Psychiatry 29:711-714

Miner CM, Davidson JR, Potts NL, Tupler LA, Charles HC, Krishnan KR (1995): Brain fluoxetine measurements using fluorine magnetic resonance spectroscopy in patients with social phobia. Biol Psychiatry 38:696-698

Palmer KJ, Benfield P (1994): Fluvoxamine. An overview of its pharmacological properties and review of its therapeutic potential in non-depressive disorders. CNS Drugs 1(1):57-87

Renshaw PF, Guimares AR, Fava M, Rosenbaum JF, Pearlman JD, Flood JG, Puopolo PR, Clancy K, Gonzalez RG (1992): Accumulation of fluoxetine and norfluoxetine in human brain during therapeutic administration. Am J Psychiatry 149:1592-1594

Strauss WL, Layton ME, Hayes CE, Dager SR (1997): ${ }^{19} \mathrm{~F}$ magnetic resonance spectroscopy investigation in vivo of acute and steady state brain fluvoxamine levels in obsessive-compulsive disorder. Am J Psychiatry 154 (4):516-522

Strauss WL, Layton ME, Dager SR (1998): Brain elimination half-life of fluvoxamine measured by ${ }^{19} \mathrm{~F}$ magnetic resonance spectroscopy. Am J Psychiatry 155(3):380-384

Weiss G, Ferretti J (1983): Accuracy and precision in the estimation of peak areas and NOE factors. J Magn Res 55:397-407

Wilde MI, Plosker GL, Benfield P (1993): Fluvoxamine. An updated review of its pharmacology, and therapeutic use in depressive illness. Drugs 46(5):895-924 